Case
Reports

Amol Raizada, MD Nachiket Apte, MD Scott Pham, MD

Key words: Antibacterial agents/therapeutic use; aortic valve/surgery; endocarditis, bacterial/epidemiology; heart valve diseases/ complications/surgery; $Q$ fever/complications/diagnosis/prevention \& control/ surgery/therapy

From: Department of Cardiovascular Diseases, Sanford School of Medicine, University of South Dakota, Sioux Falls, South Dakota 57105

Dr. Apte is now a resident in internal medicine at Johns Hopkins University/Sinai Hospital of Baltimore, Baltimore, Maryland.

Address for reprints: Amol Raizada, MD, Department of Cardiovascular Diseases, Sanford School of Medicine, University of South Dakota, 1301 W. 18th St., Sioux Falls, SD 57105

E-mail: raizadaa@gmail.com

(C) 2016 by the Texas Heart ${ }^{\circledR}$ Institute, Houston

\section{Q Fever Endocarditis}

\author{
Presenting with Superior Mesenteric \\ Artery Embolism and Renal Infarction
}

$Q$ fever is a zoonotic disease with a reservoir in mammals, birds, and ticks. Acute cases in human beings can be asymptomatic, or they can present with a flu-like illness, pneumonia, or hepatitis. Approximately 5\% of cases progress to chronic Q fever. Endocarditis, the most typical manifestation of chronic $Q$ fever, is usually associated with small vegetations that occur in patients who have had prior valvular damage or who are immunocompromised. We present what we think is the first reported case of superior mesenteric artery embolism from $Q$ fever endocarditis of the aortic valve, in a 39-year-old woman who needed surgical embolectomy and subsequent aortic valve replacement. (Tex Heart Inst J 2016;43(1):91-3)

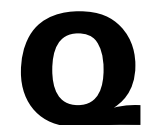
fever (QF) is a zoonotic disease that is caused by Coxiella burnetii. Patients with acute infection can be asymptomatic or can present with a flu-like illness, pneumonia, or hepatitis. Approximately 5\% of patients develop chronic QF. ${ }^{1}$ Endocarditis is the usual manifestation of chronic QF and occurs in patients who have had prior valvular damage or who are immunocompromised. Cardiac vegetations are usually small and are seen in only $12 \%$ of patients with QF endocarditis. ${ }^{2}$ Antibiotic therapy is recommended for at least 18 months in these cases, and valve surgery is reserved only for patients with hemodynamic compromise from valvular dysfunction. We present a case of $\mathrm{QF}$ endocarditis associated with a large aortic valve $(\mathrm{AV})$ vegetation with embolism to the superior mesenteric artery (SMA) and renal artery.

\section{Case Report}

In March 2013, a 39-year-old woman presented with worsening right-sided flank and abdominal pain a day after she had begun taking levofloxacin for a presumed urinary tract infection. Her medical history was significant for bacterial endocarditis 8 years earlier, nonischemic cardiomyopathy, hypertension, dyslipidemia, and psychological depression. The patient had a 10-pack-year history of smoking but disclaimed alcohol or illicit-drug use. She worked at the laundry in a local hospital and had pet cats and dogs. She reported no exposure to farm animals or to unpasteurized milk.

Physical examination revealed right-flank and upper abdominal tenderness. Laboratory findings revealed leukocytosis, and a urinalysis yielded 11 to 30 red blood cells and 0 to 5 white blood cells per high-power field. Contrast computed tomograms of the abdomen and pelvis showed splenomegaly and acute infarction of the right kidney. Radiodense structures in the right renal artery and SMA raised suspicions of calcific emboli (Fig. 1). The patient was taken for immediate abdominal exploration; SMA balloon thromboembolectomy was performed, with retrieval of calcified embolic tissue.

A transesophageal echocardiogram (TEE) showed a heterogeneous, $1.4 \times 0.5-\mathrm{cm}$ mobile mass attached to the right cusp of the AV (Fig. 2) and associated moderate, eccentric aortic regurgitation (Fig. 3). Prior serial transthoracic echocardiograms (TTE), obtained because of the patient's history of bacterial endocarditis, had revealed a small but stable nodule on the same aortic cusp. The patient was started on vancomycin, gentamicin, and ceftriaxone.

The patient underwent AV replacement with use of a 21-mm MITROF LOW pericardial valve (SoRIN S.p.A.; Milan, Italy). Blood cultures drawn at presentation and tissue cultures from the AV were negative for any growth. Histologic sections of the native AV showed calcific nodularity with organizing fibrin and associated acute inflam- 


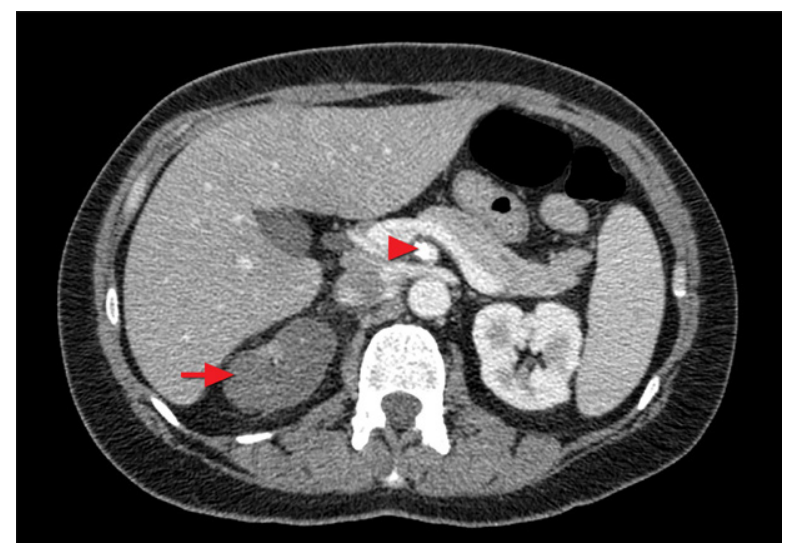

Fig. 1 Computed tomogram of the abdomen and pelvis (with contrast medium) shows an acute right renal infarction (arrow) and a calcific, partially occlusive structure in the superior mesenteric artery (arrowhead).

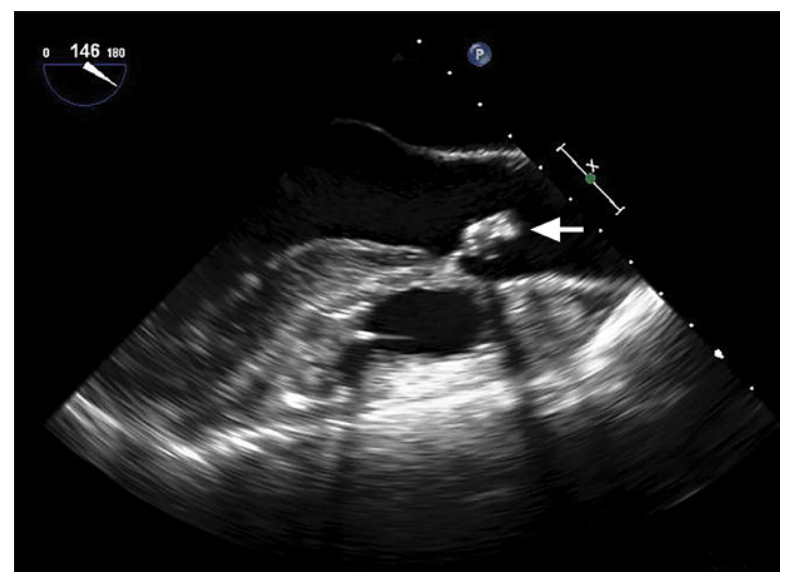

Fig. 2 Transesophageal echocardiogram (midesophageal longaxis view) shows a large vegetation on the right cusp of the aortic valve (arrow).

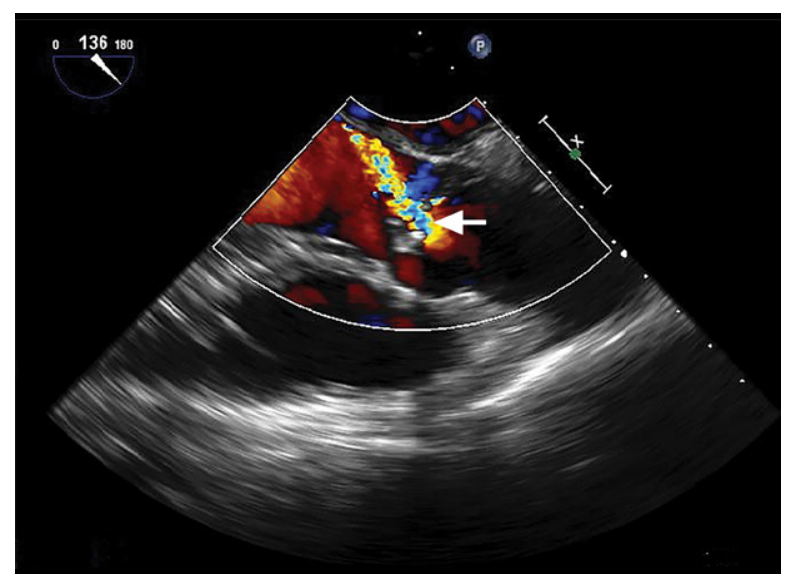

Fig. 3 In color-flow Doppler mode, transesophageal echocardiogram (midesophageal long-axis view) shows moderate eccentric aortic regurgitation (arrow) across the aortic valve. mation consistent with endocarditis. Q fever serologic investigation by means of enzyme-linked immunosorbent assay (ELISA) was positive for phase I and phase II immunoglobulin (Ig) G, IgA, and IgM antibodies at dilutions $\geq 1: 1,024$, suggesting QF endocarditis. After an 8-day hospital stay, the patient was discharged on a long-term regimen of oral doxycycline and hydroxychloroquine. At her 2-year follow-up examination in March 2015, she had no evidence of endocarditis on TTE.

\section{Discussion}

Q fever is caused by Coxiella burnetii, an obligate intracellular bacterium that is found worldwide and that accounts for $3 \%$ to $5 \%$ of all infective endocarditis cases. It was first described in 1935 in Queensland, Australia, during an outbreak of a febrile illness (query fever) among slaughterhouse workers. The reservoir of this zoonosis includes mammals, birds, and ticks. Transfer from animals to human beings usually occurs through infected aerosolized secretions. ${ }^{2}$

Acute QF presents with flu-like symptoms, hepatitis, or pneumonia after an incubation period of 2 to 3 weeks, although as many as half of cases are asymptomatic. About $5 \%$ of affected patients progress to chronic QF. ${ }^{1}$ Endocarditis, the typical manifestation of chronic $\mathrm{QF}$, can present months to years after the acute infection. Other organ systems affected by chronic QF include the vasculature, bones, and liver. ${ }^{1}$

Endocarditis usually occurs in patients who have prior valvular damage or who are immunocompromised. ${ }^{3}$ Affected patients typically present with symptoms of heart failure or valvular dysfunction. Immune-complex glomerulonephritis, as well as systemic embolism to the brain, spleen, and kidneys, might also be seen. Cardiac vegetations are usually small and are seen in only $12 \%$ of cases. ${ }^{2}$

Serologic analysis is the primary mode of diagnosing QF, because culture and DNA-amplification techniques have low sensitivities and are available only in reference laboratories. The diagnosis of chronic QF requires a phase I IgG antibody titer $\geq 1: 1,024$ and an identifiable nidus of infection. ${ }^{4}$

Q fever endocarditis needs treatment with doxycycline and hydroxychloroquine for at least 18 months in infected native valves, and for 24 months in prosthetic valves. Upon completion of therapy, the antibiotics can be discontinued if the patient exhibits clinical improvement in addition to a fourfold decrease in phase I IgG levels and the complete disappearance of phase II IgM titers. If the serologic findings do not indicate adequate improvement, consultation with a QF expert is recommended. ${ }^{4}$ Even after long-term therapy, recurrences are common. Because the cardiac vegetations are usually small, valvular surgery is reserved for patients who 
have hemodynamic compromise associated with valvular dysfunction. ${ }^{2}$

Our patient had a history of valvular damage from bacterial endocarditis, which predisposed her to QF endocarditis. She had a large vegetation (not usual in QF endocarditis) and an associated catastrophic embolic event, which necessitated SMA embolectomy and AV replacement.

\section{References}

1. Fournier PE, Marrie TJ, Raoult D. Diagnosis of Q fever. J Clin Microbiol 1998;36(7):1823-34.

2. Maurin M, Raoult D. Q fever. Clin Microbiol Rev 1999;12 (4):518-53.

3. Fenollar F, Fournier PE, Carrieri MP, Habib G, Messana T, Raoult D. Risks factors and prevention of $Q$ fever endocarditis. Clin Infect Dis 2001;33(3):312-6.

4. Anderson A, Bijlmer H, Fournier PE, Graves S, Hartzell J, Kersh GJ, et al. Diagnosis and management of Q fever--United States, 2013: recommendations from CDC and the Q Fever Working Group [published erratum appears in MMWR Recomm Rep 2013;62(35):730]. MMWR Recomm Rep 2013; 62(RR-03):1-30. 